\title{
Cathodoluminescence of Polymeric Materials
}

\author{
Anahita Pakzad ${ }^{1}$, David J. Stowe ${ }^{2}$, Steve Nagy ${ }^{1}$, Jason R. Mantei ${ }^{3}$, and John-Bruce Green ${ }^{3}$ \\ 1. Gatan Inc. R\&D Headquarters, Pleasanton, CA, USA. \\ 2. Gatan UK, Abingdon Oxon, UK. \\ 3. Baxter Healthcare, Technology Resources/Particles and Interfaces, Round Lake, IL, USA.
}

Composite materials are commonly used because, compared to their individual components, they have improved qualities such as higher strength, lighter weight, and lower production price. Two of the most important factors affecting the final properties of such materials are the composition and size/spatial distributions of their individual components. These properties have been studied using various microscopy techniques such as atomic force microscopy (AFM), transmission electron microscopy (TEM) and scanning electron microscopy (SEM).

When an SEM is available, some of the most common techniques are: Backscatter Electron Imaging (based on atomic number contrast), X-ray Computed Tomography (based on phase and absorption contrast) and Energy Dispersive X-ray Spectroscopy (based on elemental characteristic X-rays). One common limiting factor of all of these techniques is that in the case of organic materials (mostly made of light elements such as carbon, hydrogen and oxygen), acquisition of images with sufficient contrast and signal/noise ratio can be challenging. In such cases, an alternative technique that can differentiate materials independent of their elemental composition is necessary.

Cathodoluminescence (CL) analysis inside an SEM is a well-established analytical technique used in many industrial and academical laboratories to study minerals, glasses, ceramics, gemstones, semiconductors, rare earths and optoelectronic materials. In such experiments, the primary electron beam excites electrons in a crystal or a molecule ground state. When these excited electrons go back to their original state (through electron-hole pair recombination), photons with energy corresponding to this transition energy $(0.3$ to $6 \mathrm{eV})$ are emitted. These photons are then used for imaging and spectroscopy to gather information on the crystallographic and chemical properties of these materials at the microscopic level.

In the current study, we used a Gatan MonoCL4 installed on a Zeiss Sigma VP SEM to study the change in CL emission of various types of polymers. Experiments were performed at room temperature, using a $12 \mathrm{kV}$ electron beam, at variable pressure mode (20 $\mathrm{Pa}$, to reduce charging artifacts). The spectra and images were collected using a $150 \mathrm{1} / \mathrm{mm}$ diffraction grating and a CCD or a photo multiplier tube (PMT), respectively. Luminescence is exhibited by many organic compounds which possess aromatic molecules or molecules having conjugated double bonds. We observed significant spectral differences between the studied polymers and demonstrated that these differences can be used as a means to distinguish them in a compound system. Such experiments can be applied in a large scale to assess the homogeneity of polymer compounds and to improve and optimize their manufacturing processes. 


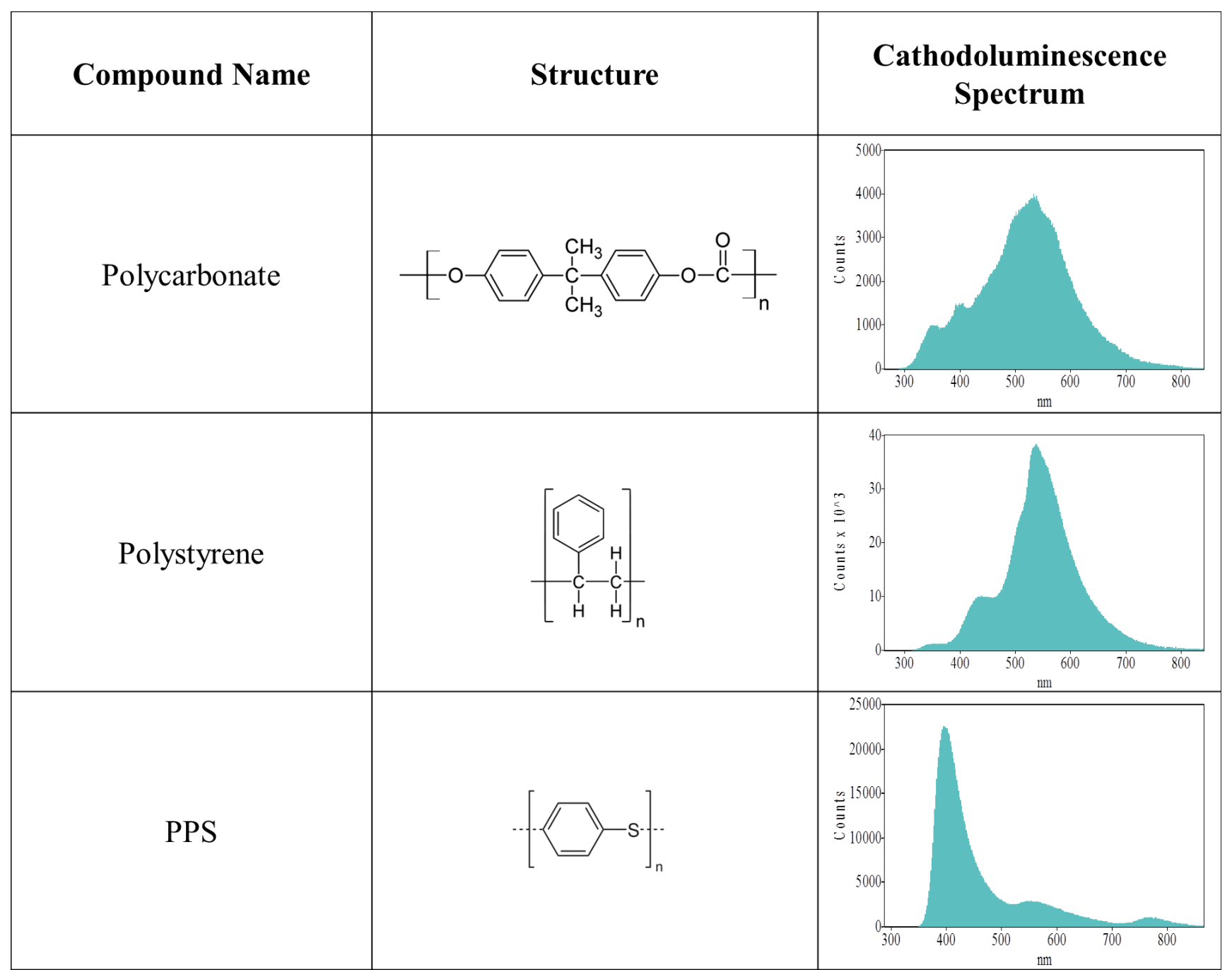

Figure 1. Example of CL spectra acquired from three polymers. The vertical and horizontal axes in the spectra show the CL counts and wavelength (nm), respectively. 